\title{
\begin{tabular}{l|l} 
Mitraries & DSpace@MIT
\end{tabular}
}

\author{
MIT Open Access Articles
}

Oversampling transmit and receive antenna arrays

The MIT Faculty has made this article openly available. Please share how this access benefits you. Your story matters.

Citation: Wornell, Gregory W. et al. "Oversampling transmit and receive antenna arrays." Proceedings of the IEEE International Conference on Acoustics, Speech, and Signal Processing (ICASSP), 2010: 2522-2525. (C) 2010 IEEE.

As Published: http://dx.doi.org/ 10.1109/ICASSP.2010.5496305

Publisher: Institute of Electrical and Electronics Engineers (IEEE)

Persistent URL: http://hdl.handle.net/1721.1/73551

Version: Final published version: final published article, as it appeared in a journal, conference proceedings, or other formally published context

Terms of Use: Article is made available in accordance with the publisher's policy and may be subject to US copyright law. Please refer to the publisher's site for terms of use. 


\section{OVERSAMPLING TRANSMIT AND RECEIVE ANTENNA ARRAYS}

\author{
Chen-Pang Yeang \\ IHPST, Univ. Toronto, ON, Canada \\ chenpang.yeang@utoronto.ca
}

\begin{abstract}
A dense antenna array architecture is developed to ease the circuit requirements of the radio frequency $(\mathrm{RF})$ front-end in beamforming applications. In the architecture, antennas are spaced more closely than would otherwise be required to exploit the available degrees of freedom. Such an array structure is analogous to temporally oversampled data conversion systems, which have reduced quantizer resolution requirements. For a linear, uniformly-spaced array, we develop a spatial-domain version of $\Delta \Sigma$ quantization, and show that with binary quantization for the in-phase and quadrature components of antenna weights, even relatively modest amounts of oversampling can reproduce beamforming patterns of interest to practically useful levels of accuracy.
\end{abstract}

Index Terms - Phased-arrays antennas, $\Delta \Sigma$ quantization, array processing, RF circuits, millimeter-wave imaging, wireless communication

\section{INTRODUCTION}

Recent progress in millimeter-wave and microwave integrated circuit (IC) manufacturing technology has created new opportunities for the development of novel, low-cost antenna array architectures. Indeed, the ability to integrate miniature microstrip antennas has expanded the realm of applications for antenna arrays to a variety of embedded communication and sensing systems. In such systems, the associated specifications on RF components such as phase-shifters, oscillators, and amplifiers, can be challenging to meet in a cost-effective manner. In this paper, we develop a dense antenna array to ease such RF circuit requirements without sacrificing performance, and demonstrate its effectiveness for transmit and receive beamforming in phasedarray applications.

\section{THE TRADITIONAL ARRAY ARCHITECTURE}

In a traditional uniform linear array, an aperture of length $L$ is formed from a collection of antenna elements, adjacent pairs of which are spaced $d$ apart. The nominal spacing between elements is $d=\lambda / 2$. Provided that elements are at least as close as this nominal spacing, the maximum number of available degrees of freedom offered by the aperture can be realized, and, in phased-array applications in particular, grating lobes in beamforming patterns can be avoided. Thus, since $L=(N-1) d$, the minimum number of antenna elements required is typically $N_{\circ}=1+2 L / \lambda$.

With the associated antenna array architecture, depicted in Fig. 1, a beam pattern can be formed by choosing antenna element

This work was supported in part by DARPA under Contract No. HR0011-06-1-0004, and by C2S2 in the FCRP of SRC.

\author{
Gregory W. Wornell and Lizhong Zheng \\ Dept. EECS, MIT, Cambridge, MA, USA \\ $\{$ gww,lizhong $\} @$ mit.edu
}

weights $w_{n}=a_{n} e^{j \theta_{n}}$. This is implemented via $N$ amplitude attenuators and $N$ phase shifters. At the transmitter, the RF signal passes through such a beamformer, whose $N$ outputs are directed to $N$ power amplifiers and then $N$ antennas. At the receiver, the $N$ antenna signals that result from the incoming wave pass through $N$ low-noise amplifiers, are phase-shifted, amplitude-adjusted, and superimposed via the beamformer to form the output signal. The associated transmitter and receiver array radiation patterns are, respectively,

$B^{\mathrm{T}}(\psi)=\left|\sum_{n=1}^{N} w_{n} e^{-j n k d \cos \psi}\right|, \quad B^{\mathrm{R}}(\psi) \equiv\left|\sum_{n=1}^{N} w_{n} e^{j n k d \cos \psi}\right|$,

where $k=2 \pi / \lambda$ is the wave number and $\psi$ is the target angle of radiation. Note that the narrowest beam that can be formed by such processing has main lobe width proportional to $\lambda / L$ when $N>N_{\circ}$, where the constant of proportionality does not depend on $N$. Additional antenna elements do not further improve the array's resolution, regardless of how the $w_{n}$ are chosen [1].

The classical array architecture poses challenges to circuit design and device technology. Accuracy of the array radiation patterns in (1) depends on the accuracy with which $\left\{\theta_{n}\right\}$ and $\left\{a_{n}\right\}$ are implemented. This implies that the phase shifters in the beamformers must be implemented with high resolution. Moreover, the power amplifiers at the transmitter must have high linearity.(Linearity is less a problem for low-noise amplifiers at the receiver as they are less likely to get saturated by the weak input.) Although a variety of approaches have been proposed to improve the precision of these components, most remain expensive and complicated; see, e.g., [2-7]. For example, phase shifting might be accurately performed at the baseband with all-digital circuitry. But in such a scheme, all the array signals have to be up-converted to RF band after phase-shifting (at the transmitter) or undergo down-conversion from RF before phase shifting (at the receiver). This means the phase relations between the array signals must be preserved through up-conversion or downconversion, which imposes challenging phase noise requirements on the associated mixers

\section{AN OVERSAMPLING ARRAY ARCHITECTURE}

In contrast with the traditional architecture, we consider packing more antennas $N$ in the given aperture than would otherwise be required, i.e., $N>N_{\circ}$, for both transmit and receive array configurations. This density is then exploited in a manner analogous to the way temporal oversampling is exploited in data converter design. In particular, just as sampling at a rate exceeding that dictated by the Nyquist criterion reduces quantization resolution requirements, the dense array uses $N$ lower-quality RF components instead of $N$ 。 higher quality ones. 


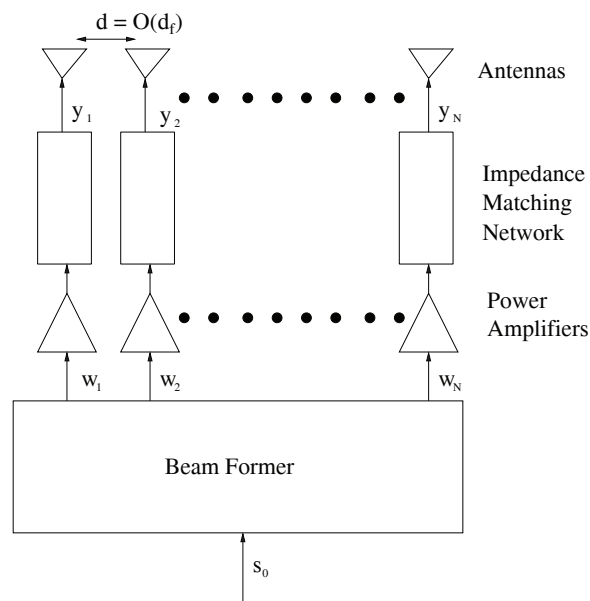

(a) Transmitter

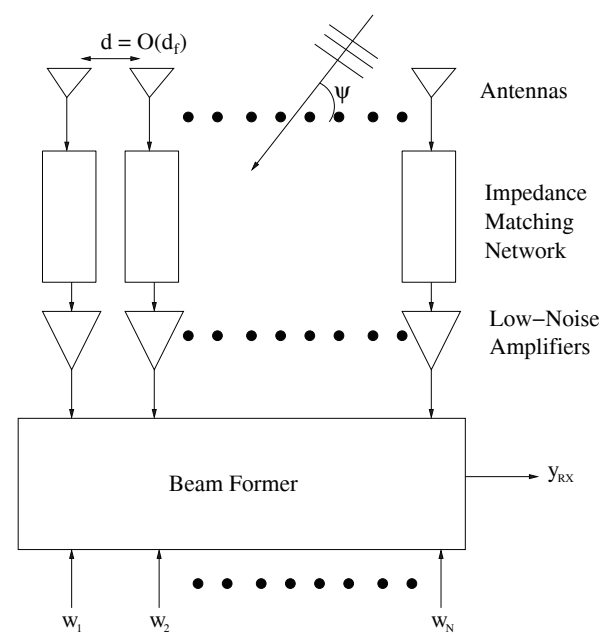

(b) Receiver

Fig. 1. The classical antenna array architecture.

\subsection{Spatial $\Delta \Sigma$ Quantization}

To reduce our RF component specifications, we constrain our system to use coarsely quantized versions of the weights $w_{n}$, and exploit the oversampling to minimize the effect of such quantization. In particular, we exploit the principle of $\Delta \Sigma$ quantization [8] by directly translating it to the spatial domain. (For other rather different multidimensional extensions of $\Delta \Sigma$ quantization motivated by image processing and space-time coding, see, e.g., [9-11].)

The spatial $\Delta \Sigma$ architecture is depicted in Fig. 2, and involves creating a sequence of quantized weights $v_{n}$ that "act" in a manner asympotically indistinguishable from the desired weights $w_{n}$. The associated processing proceeds as follows. At the $n$th antenna, we obtain the difference between the desired weight $w_{n}$ and its counterpart $v_{n}$ at the output of the corresponding quantizer, corresponding to the $\Delta$ part of the structure. We then add that difference to the input of the $n$th quantizer, corresponding to the $\Sigma$ part of the structure. The result is then quantized using 4-PSQ (phase-shift quantization, where the input is quantized to a constant amplitude and one of four phases: $0^{\circ}, 90^{\circ}, 180^{\circ}, 270^{\circ}$ ), which corresponds to quantizing each of the in-phase and quadrature components to a single bit. As such

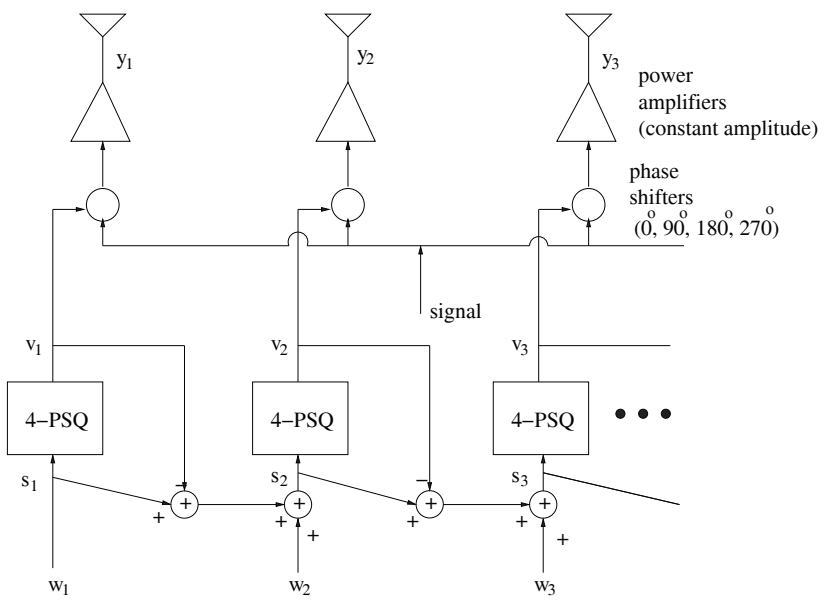

Fig. 2. The spatial $\Delta \Sigma$ architecture at the transmitter. The corresponding receiver architecture is obtained by reversing the beamformer to have multiple inputs and a single output, i.e., the $n$th antenna input from the low-noise amplifier is mixed with $v_{n}$, then all such terms are summed.

only crude 4-angle phase shifters and (nonlinear) bilevel power amplifiers are required in the RF front-end.

\section{RESULTS}

In this section, we sketch some of the associated analysis of the dense-antenna architecture, present some simulation results, and discuss some implementation issues.

\subsection{Analysis}

Spatial $\Delta \Sigma$ oversampling can be understood in a manner analogous to the way traditional temporal $\Delta \Sigma$ oversampling is understood. In the latter, a time series $\left\{w_{n}\right\}$ is quantized into another time series $\left\{v_{n}\right\}$, from which one can recover $\left\{w_{n}\right\}$ by low-pass filtering. That recovery is possible follows from the fact that the feedback structure of $\Delta \Sigma$ forces the average value of the quantized output to track the average input, which has the advantage of suppressing the quantization error spectrum at low frequencies. Similar behavior takes place in the case of our spatial $\Delta \Sigma$ scheme. To see how the associated spatial low-pass filtering arises, note that the transmit and receive array patterns $B^{\mathrm{T}}(\psi)$ and $B^{\mathrm{R}}(\psi)$ in (1) are effectively the Fourier transforms of the weights $\left\{w_{n}\right\}$ or $\left\{v_{n}\right\}$ at the spatial frequency $\omega= \pm k d \cos \psi= \pm k L \cos \psi /(N-1)$. Hence, $\omega \rightarrow 0$ as $N \rightarrow \infty$, i.e., the beamforming operation itself provides the required spatial low-pass filtering.

Ignoring mutual coupling between antennas for the moment, one can show formally that (for transmit arrays)

$$
\hat{B}^{\mathrm{T}}(\psi)-B^{\mathrm{T}}(\psi)=O\left(\frac{1}{\sqrt{N}}\right)
$$

where

$$
\hat{B}^{\mathrm{T}}(\psi)=\left|\sum_{n=1}^{N} v_{n} e^{-j n k d \cos \psi}\right| .
$$

The corresponding receive array expressions $\hat{B}^{\mathrm{R}}(\psi)-B^{\mathrm{R}}(\psi)$ are obtained by replacing $j$ with $-j$. 
A rough sketch of the derivation of (2) is as follows, and mirrors the familiar $\Delta \Sigma$ analysis. The transmitter architecture illustrated in Fig. 2 leads to

$$
v_{n}=w_{n}+\tilde{e}_{n}-\tilde{e}_{n-1},
$$

where $\tilde{e}_{n}$ is the quantization error. Substituting the above $v_{n}$ into (3), we obtain

$$
\begin{aligned}
& \hat{B}^{\mathrm{T}}(\psi)=\mid \sum_{n=1}^{N} e^{-j n k d \cos \psi} w_{n} \\
&+\left(1-e^{-j k d \cos \psi}\right) \sum_{n=1}^{N} e^{-j n k d \cos \psi} \tilde{e}_{n} \\
&+e^{-j(N+1) k d \cos \psi} \tilde{e}_{N} \mid
\end{aligned}
$$

The first term in (4) has magnitude equal to $B^{\mathrm{T}}(\psi)$. For large $N$, the contribution from the third term in (4) is negligible, and thus to verify that $\hat{B}^{\mathrm{T}}(\psi)$ approaches $B^{\mathrm{T}}(\psi)$ in the limit of large $N$, it suffices to argue that the second-term in (4) also vanishes asymptotically. To this end, we have

$$
\begin{aligned}
& N\left\langle\left|1-e^{-j k d \cos \psi}\right|^{2} \sum_{n, m=1}^{N} e^{-j(n-m) k d \cos \psi} \tilde{e}_{n} \tilde{e}_{m}^{*}\right\rangle \\
& =N\left|1-e^{-j k d \cos \psi}\right|^{2} \sum_{n, m=1}^{N} e^{-j(n-m) k d \cos \psi}\left\langle\tilde{e}_{n} \tilde{e}_{m}^{*}\right\rangle \\
& =\left(1-e^{-j k d \cos \psi}\right)\left(1-e^{j k d \cos \psi}\right) N^{2} \sigma_{e}^{2} \\
& \rightarrow k^{2} \cos ^{2} \psi L^{2} \sigma_{e}^{2} \text { as } N \rightarrow \infty,
\end{aligned}
$$

where we have used that the quantization errors are uncorrelated, i.e., $\left\langle\tilde{e}_{n} \tilde{e}_{m}^{*}\right\rangle=\sigma_{e}^{2} \delta_{n, m}$ and $d=L /(N-1)$. A very similar argument yields the associated receive array result.

The above analysis does not consider the effect of mutual coupling between antennas in the array. In additional analysis, we incorporate mutual coupling effects on the array radiation patterns by deriving from the S-parameter formulation modifications of (1) involving the array's impedance matrix. And we compute all entries of the impedance matrix via the induced EMF method $[12,13]$.

\subsection{Simulation}

Figs. 3 and 4 show the simulated array pattern error $\|\hat{B}-B\|$ as a function of the oversampling ratio $N / N$ 。 without and with mutual coupling, respectively, in the case of a phased array. The (normalized) array pattern errors are averaged over the radiation angle $\psi$ and array weights $w_{n}=e^{j n \theta_{0}}$ with various $\theta_{0}$. The wavelength is $\lambda=3$ $\mathrm{cm}$ and the overall array aperture is $L=5 \lambda=15 \mathrm{~cm}$. In the computation of mutual coupling, the antennas are modeled as cylindrical radiators with radius $1 \mathrm{~mm}$ and length $1 \mathrm{~cm}$. As Fig. 3 reflects, the quantization error of array patterns generally decays with the number of antennas, ultimately approaching the $1 / \sqrt{N}$ asymptote.

Note that the curve is not smooth. This is likely due to the fact that we do not take samples of antenna weights $\left\{w_{n}\right\}$ over many values. We only restrict the possibilities to the phased-array cases $w_{n}=e^{j n \theta_{0}}$, which are the most relevant to beamforming. Moreover, we only average over $N_{\circ}$ distinct values of $\theta_{0}$. Thus when $N$ is small, the total number of data points (over distinct $n$ and $\theta_{0}$ ) are really too few to warrant the law of large numbers and accordingly to smooth the quantization errors. Increasing the number of

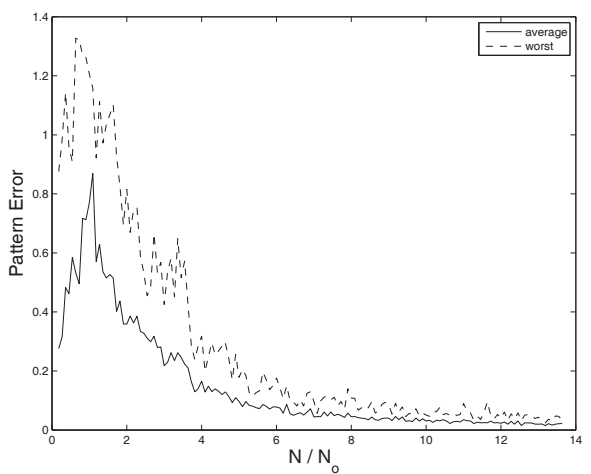

Fig. 3. The average and worst quantization errors over various radiation patterns (without mutual coupling); phased-array inputs, $\lambda=3$ cm, $L=5 \lambda$.

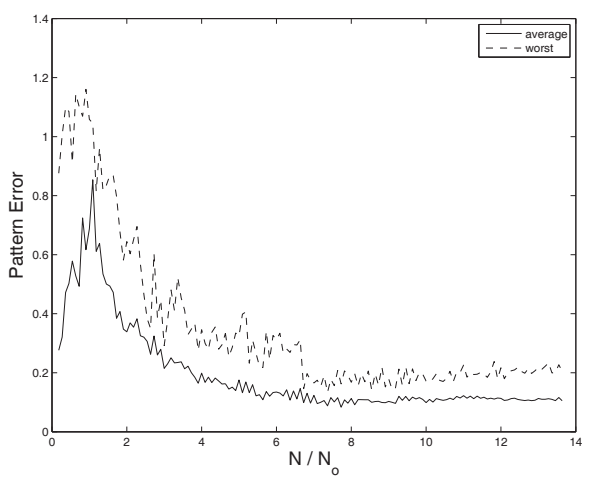

Fig. 4. The average and worst quantization errors over various radiation patterns (with mutual coupling); phased-array inputs, $\lambda=3$ $\mathrm{cm}, L=5 \lambda$; cylinder antennas with length $1 \mathrm{~cm}$ and radius $1 \mathrm{~mm}$.

$\theta_{0}$ within the range of $[0, \pi]$ does not significantly smooth the curve, either, for the beam patterns become highly overlapped and hence correlated with one another as the number of samples of $\theta_{0}$ exceeds $N_{\circ}$.

Fig. 4 shows that antenna mutual coupling does incur some penalty in convergence rate of the beam pattern quantization error. While a detailed analysis is ongoing, we have found that the mutual coupling effect on pattern errors can be reduced to a multiplicative factor at large $N$, owing to the antenna impedance matrix's Toeplitz structure, i.e., $B^{\text {coupled }}(\psi) \approx C(\psi) B^{\text {uncoupled }}(\psi)$. Our calculations indicate that $C(\psi)$ slowly converges to a fairly flat pattern as $N$ becomes large. In any case, the benefits of oversampling are apparent in this analysis as well.

Fig. 5 compares the actual radiation patterns of ideal phased arrays and $\Delta \Sigma$ quantized phased arrays with mutual coupling. Two particular values of oversampling ratio are chosen: (a) $N / N_{\circ}=1.5$, and (b) $N / N_{\circ}=11.6$. Each plot shows seven distinct ideal phasedarray patterns with roughly non-overlapping mainlobes and the corresponding quantized, mutually coupled patterns. As is apparent, the quantized patterns closely approximate the target patterns even for modest amounts of spatial oversampling. 


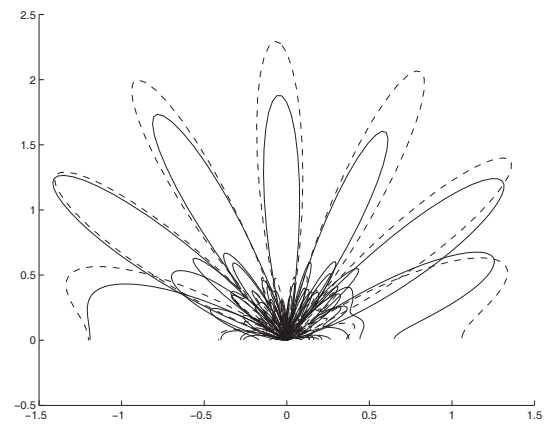

(a) $N / N_{\circ}=1.5$

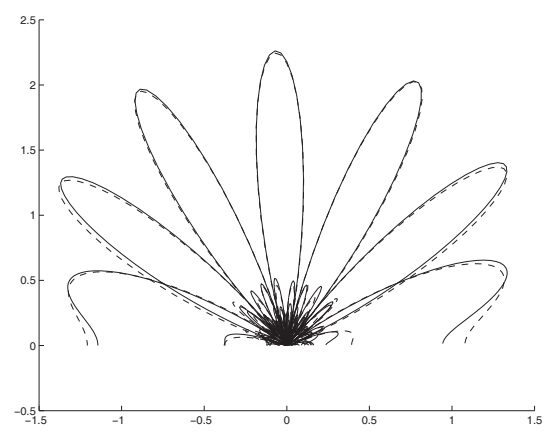

(b) $N / N_{\circ}=11.6$

Fig. 5. Radiation beam patterns of ideal phased arrays (dashed curves) and $\Delta \Sigma$ quantized arrays (solid curves), with mutual coupling taken into account.

\subsection{Implementation Issues}

Recent progress in the millimeter-wave integrated circuit (MMIC) technology makes it increasingly practical to implement the dense array system. The most serious manufacturing challenge of the system is to make a closely separated antenna array within a limited aperture. Fortunately, the availability of various mini-scaled, embedded radiation elements, such as microstrip antennas and on-chip dipole antennas, has resolved much of the difficulty associated with implementing the dense antenna array. In the $77 \mathrm{GHz}$ phased-array transceiver with on-chip dipole antennas on silicon substrate in [14, 15], for instance, the separation between two antennas is less than $1.7 \mathrm{~mm}$. By contrast, in our numerical cases for the $10 \mathrm{GHz}$ dense array in which the aperture size is $15 \mathrm{~cm}$ and the number of elements is less than 150 , the minimum inter-antenna distance is $1 \mathrm{~mm}-\mathrm{a}$ value with the same order of magnitude as that of the actually implemented $77 \mathrm{GHz}$ transceiver. The dozens of radiation elements in an electronically tunable reflectarray in [16] also indicates the possibility of implanting and controlling a large number of patched antennas on a confined surface.

The other parts of the system (not discussed in this paper) are also practically implementable. The impedance matching network can be made with simple components such as reactors and quarterwave transmission lines that do not require tuning and do not couple energy between distinct signal paths. The RF devices, mixers, and $\Delta \Sigma$ quantizers can be embedded in existing integrated circuitry.

\section{REFERENCES}

[1] D. H. Staelin, Receivers, Antennas, and Signals (course notes), No. 20, "Basic radar ambiguity," MIT OpenCourseWare.

[2] J.-S. Fu and A. Mortazawi, "Improving power amplifier efficiency and linearity using a dynamically controlled tunable matching network," IEEE Trans. Microwave Theory, Techniques, vol. 56, , pp. 3239-3244, Dec. 2008.

[3] S. H. Ji, S. K. Eun, C. S. Cho, J. W. Lee, and J. Kim, "Linearity improved Doherty power amplifier using composite right/lefthanded transmission lines," IEEE Microwave, Wireless Components Lett., vol. 18, pp. 533-535, Aug. 2008.

[4] A. van Bezooijen, R. Mahmoudi, and A. H. M. van Roermund, "Adaptive methods to preserve power amplifier linearity under antenna mismatch conditions," IEEE Trans. Circuits, Syst. I, vol. 52, pp. 2101-2108, Oct. 2005.

[5] D. Parker and D. C. Zimmermann, "Phased arrays-Part II: Implementations, applications, and future trends," IEEE Trans. Microwave Theory, Techniques, vol. 50, pp. 688-698, Mar. 2002.

[6] J. Roderick, H. Krishnaswamy, K. Newton, and H. Hashemi, "Silicon-based ultra-wideband beam-forming," IEEE J. SolidState Circuits, vol. 41, pp. 1726-1739, Aug. 2006.

[7] H. Hashemi, X. Guan, A. Komijani, and A. Hajimiri, "A 24$\mathrm{GHz}$ SiGe phased-arry receiver-LO phase-shifting approach," IEEE Trans. Microwave Theory, Techniques, vol. 53, pp. 614626, Feb. 2005.

[8] S. R. Norsworthy, R. Schreier, and G. C. Temes, Delta-Sigma Data Converters: Theory, Design, and Simulation, New York: IEEE Press, 1997.

[9] R. Floyd and L. Steinberg, "An adaptive algorithm for spatial grayscale,” Proc. Soc. Image Display, vol. 17, pp. 75-77, 1976.

[10] T. D. Kite, B. L. Evans, A. C. Bovik, and T. L. Sculley, "Digital halftoning as 2-D delta-sigma modulation," Proc. ICIP, 1997, pp. 799-802.

[11] D. P. Scholnik and J. O. Coleman, "Space-time vector deltasigma modulation," Proc. IEEE Radar Conf., 2004, pp. III719-722.

[12] R. W. P. King, G. J. Fikioris, and R. B. Mack, Cylindrical Antennas and Arrays, Cambridge: Cambridge University Press, 2002.

[13] J. D. Kraus, Antennas, New York: McGraw-Hill, 1950.

[14] A. Babakhani, X. Guan, A. Komijani, A. Natarajan, and A. Hajimiri, "A 77-GHz phased-array transceiver with on-chip antennas in silicon: receiver and antennas," IEEE J. Solid-State Circuits, vol. 41, pp. 2795-2806, Dec. 2006.

[15] A. Natarajan, A. Komijani, X. Guan, A. Babakhani, and A. Hajimiri, "A 77-GHz phased-array transceiver with on-chip antennas in silicon: transmitter and local LO-path phase shifting," IEEE J. Solid-State Circuits, vol. 41, pp. 2807-2818, Dec. 2006.

[16] S. V. Hum, M. Okoniewski, and R. J. Davies, "Design and modeling of electronically tunable reflectarrays," IEEE Trans. Antennas, Propag., vol. 55, pp. 2200-2210, 2007. 